\title{
Evidence for changes in behaviour leading to reductions in HIV prevalence in urban Malawi
}

\author{
George Bello, ${ }^{1,2}$ Bertha Simwaka, ${ }^{2}$ Tchaka Ndhlovu, ${ }^{2}$ Felix Salaniponi, ${ }^{1}$ \\ Timothy B Hallett ${ }^{3}$
}

- An additional table is published online only. To view this file please visit the journal online (http://sti.bmj.com).

${ }^{1}$ Ministry of Health, Lilongwe, Malawi

${ }^{2}$ REACH Trust, Lilongwe, Malawi

${ }^{3}$ Department of Infectious Disease Epidemiology, Imperial College London, London, UK

\section{Correspondence to}

Timothy B Hallett Department of Infectious Disease

Epidemiology, Imperial College London, St Mary's Campus, Norfolk Place, London, W2 1PG; timothy.hallett@imperial.ac.uk

Accepted 13 February 2011 Published Online First 23 March 2011

\begin{abstract}
Background At the epicentre of the HIV epidemic in Eastern Africa, HIV prevalence has appeared to stabilise in most countries. However, there are indications that the HIV epidemic in Malawi has recently declined. Methods We analysed sexual behaviour survey data from Malawi between 2000 and 2004 and HIV prevalence data from the national antenatal clinic HIV surveillance system between 1994 and 2007 using a mathematical modelling technique that can identify associations between behaviour change and reductions in incidence.
\end{abstract}

Results In Malawi between 2000 and 2004 there were significant reductions in the proportion of 15-19 year olds starting sex, the proportion of men having sex with more than one woman in the previous year and significant increases in condom use by men with multiple partners. In the same period, prevalence dropped from $26 \%$ to $15 \%$ in urban areas among pregnant women and reduced by $40 \%$ among women aged $15-24$ years. In the same period, prevalence remained at $\sim 12 \%$ in rural areas. Mathematical modelling suggests that the declines in prevalence in urban areas were associated with the behaviour changes and that, if the changes are maintained, this will have cumulatively averted 140000 (95\% interval: 65000 to 160000 ) HIV infections by 2010.

Conclusion Changes in sexual behaviour can avert thousands of new HIV infections in mature generalised hyper-endemic settings. In urban Malawi, the reduction in the number of men with multiple partners is likely to have driven the reduction in incidence. Understanding the causes of this change is a priority so that successful programmes and campaigns can be rapidly expanded to rural areas and other countries in the region.

\section{INTRODUCTION}

Almost 30 years into the HIV epidemic, there is more need than ever to understand how interventions can stop the spread of HIV. Following billion dollar investments in prevention interventions by President's Emergency Plan for AIDS Relief (PEPFAR) and the Global Fund, there remains little sign of epidemics coming under control ${ }^{1}$ weakening the case for renewed investment. Indeed, the accumulation of randomised control trials that failed to show efficacy, ${ }^{2}$ coupled with most large HIV epidemics seeming to maintain steady endemic states, ${ }^{3}$ has led to 'crisis of confidence' in an ability to prevent infections through changes in behaviour. This vacuum of compelling prevention causes has been filled with optimistic projections for the impact of male circumcision interventions, ${ }^{4} 5$ pre-exposure prophylaxis ${ }^{6}$ and the suggestion that immediate initiation of anti-retroviral therapy would be a more effective way to reduce HIV transmission ${ }^{7}$ - but for HIV to be eliminated, not just reduced, these interventions would always have to be reinforced with substantial changes in behaviour.

However, arguments about the lack of success in HIV prevention through behaviour change have overlooked routine HIV surveillance data as a key source of information about the success of interventions and information about whether behaviour change can affect the course of large matured generalised epidemics. Indeed, it was through analyses of routine epidemiological surveillance data that the success of the 'zero-grazing' campaign in Uganda, 89 and the ' $100 \%$ condom' campaign in Thailand, ${ }^{10} 11$ were first detected and, more recently, where evidence for dramatic reductions in incidence in Zimbabwe was found. ${ }^{12} 13$ However, such analyses are not straightforward for two reasons: first, when epidemics mature, prevalence can decline even if individuals do not change their risk behaviour 81415 and these 'natural epidemiological dynamics' confound simple statistical tests for trends in prevalence (since a significant decline in prevalence does not necessarily indicate behaviour changes reducing infectious spread). Second, the non-linear threshold relationship between sexual risk behaviour and HIV spread means changes in sexual behaviour indicators, though apparently substantial, may not be epidemiologically relevant: for instance, if they are overwhelmed by high degrees of risk of other types, or they do not reduce the level of risk below a certain threshold or they are confined to parts of the population at little risk of acquiring of transmitting infection. ${ }^{16} 17$

Therefore, to interpret the information contained in these data it is essential to construct a mathematical model that provides a mechanistic description of the link between individual sexual behaviour and transmission in the population and that can simulate these natural epidemiological dynamics. Observed trends in prevalence data can then be compared against the model output to assess the evidence for observed changes in sexual behaviour actually having an impact on the course of the epidemic beyond what natural changes in prevalence would be expected.

In this paper we present an analysis of the epidemic in Malawi and show evidence that changes in the number of sex partners and condom use have contributed to a large reduction in HIV prevalence in urban areas. 


\section{METHODS}

Our analysis was conducted in two stages: first we analysed trends in key indicators of sexual behaviour over time and, second, we used a mathematical model to test for the association between these trends and the observed trajectory of the HIV epidemic.

\section{Analysis of trends in sexual behaviour indicators}

The best available data on sexual behaviour among the general population comes from the Malawi Demographic Health Surveys. Two surveys have been completed in Malawi in 2000 ( $N=13220$ women and 3092 men) and 2004 (N=11 698 women and $3261 \mathrm{men}){ }^{18} 19$ These use a nationally representative sample of households and administer a face-to-face survey that includes questions on key sexual behaviour indicators.

These data were used to construct the following indicators for both surveys, which correspond to three dimensions of sexual behaviour that are commonly associated with HIV risk: early sexual debut, partner numbers and condom use:

- Fraction of men/women aged 15-19 years that first had sex before aged 15 years.

- Fraction of men/women aged 15-24/15-49 years that have had sex with two or more partners in the last 12 months.

- Fraction of men/women aged 15-25/15-49 years that have had sex with two or more partners in the last 12 months that used a condom at last sex.

\section{Mathematical modelling analysis}

A mathematical model of the HIV epidemic in Malawi was parameterised with behavioural indicator data and compared to prevalence data in a Bayesian melding framework ${ }^{20} 21$ to test the hypothesis that the observed trends in prevalence partly result from observed changes in sexual behaviour.

The mathematical model represents the heterosexual transmission of HIV in a sex and sexual activity stratified sexuallyactive adult population (without detailed age structure), ${ }^{14} 22$ and is defined by a set of ordinary differential equations that are solved numerically. Full details of the model have previously been published by Hallett et $a l^{13}$ and further information is available from the authors on request.

For each model parameter, a probability distribution is defined that represents prior knowledge about likely values. Where possible, these priors are based on survey data but if there is no information for a particular parameter a non-informative prior is used. Details about the main parameters are listed in table S1 in the online material. Prior limits on simulated prevalence can also be set so that, despite there being little data available early in the epidemic, simulations in which prevalence rise too quickly or slowly can be excluded. ${ }^{21} 23$

The simulated prevalence time-series is compared to prevalence data from the national Antenatal Clinic (ANC) surveillance system. In Malawi, this system was started in 1994 and has included 28 different clinics in urban area and 26 different clinics in rural areas. In total, there are data on 70922 women (53556 in urban settings; 17366 in rural settings). However, many of these new sites were added only in 2007 and the number of sites consistently sampled between 1994 and 2007 is 11 in urban areas and 8 in rural areas. Note that these are the 'raw' data collected in the country and the changes in methods used by UNAIDS to produce their national prevalence estimates would not interfere with this analysis. ${ }^{24}$

The model is compared to these data using an approach that analyses trends in prevalence within each site ('random effects model') to avoid the possibility that declines in prevalence are spuriously indicated by the recruitment of progressively more remote clinics in the sentinel surveillance system. ${ }^{25}$ Since estimates of prevalence in the ANC data may include a systematic bias with respect to prevalence in the general population, ${ }^{22} 26$ the model-simulated prevalence time-series is recalibrated to correct for any observed discrepancy between ANC measures of prevalence and measures of prevalence in the general population in the same year. ${ }^{23} 27$

To test the hypothesis that changes in sexual behaviour have contributed to altering the natural course of the epidemic, two models are compared: one which does not allow behaviour change $\left(M_{0}\right)$ with one that does $\left(M_{1}\right)$. The Bayes factor measures the relative agreement to the data of the two models $\left(M_{1}\right.$ vs $\left.M_{0}\right)$ across all parameter space:

$$
K=\frac{\int p(\boldsymbol{\theta}) p\left(\mathbf{W} \mid \boldsymbol{\theta}, M_{1}\right) d \boldsymbol{\theta}}{\int p(\boldsymbol{\theta}) p\left(\mathbf{W} \mid \boldsymbol{\theta}, M_{0}\right) d \boldsymbol{\theta}}
$$

Here $\boldsymbol{\theta}$ denotes the model input parameters, $p(\boldsymbol{\theta})$ is the prior probability of that parameter set and $p\left(\mathbf{W} \mid \theta, M_{\mathbf{x}}\right)$ is the likelihood of the simulated epidemic given the data from antenatal clinics in the model $M_{x}$. Following convention, ${ }^{28}{ }^{29}$ we judge that there is strong evidence for behaviour change influencing the epidemic if $2 \ln (K)>5$ indicating that model $M_{1}$ is in substantially better agreement with the data. In that case, the number of infections averted is calculated by comparing two runs of model $M_{1}$ with the behaviour change parameters either set to their best-fitting values or to values indicating no behaviour change.

\section{RESULTS}

\section{Trends in sexual risk behaviour indicators}

Trends in the key sexual behaviour indicators are summarised in figure 1. The percentage of young women and men aged 15-19 years who first had sex before the age of 15 decreased in both sexes between 2000 and 2004 (figure 1A). In young men, the decrease was more substantial (reducing from 29\% in 2000 to $18 \%$ in $2004 ; p<0.001)$ whereas in young women the change was modest (reducing from $17 \%$ in 2000 to $14 \%$ in 2004; $\mathrm{p}=0.01)$.

In the same period, there was substantial decrease in proportion of men who had sex with more than two or more partners in the last 12 months: reducing from 15\% in 2000 to $9 \%$ among adult men aged $15-49$ years $(\mathrm{p}<0.001)$ and from $12 \%$ in 2000 to $7 \%$ in 2004 among 15-24 year olds $(\mathrm{p}<0.001)$ (figure 1B). Only $1 \%$ of women reported having two or more partners and this did not change between the two surveys.

The proportion of men with two or more partners that used condoms increased between 2000 and 2004: from 14\% in 2000 to $20 \%$ in 2004 among 15-49 year old men $(p=0.04)$ and, not statistically significantly, from $27 \%$ in 2000 to $35 \%$ in 2004 among 15-24 year old men $(p=0.2)$. There was no change in the proportion of women reporting using condoms, but the number of women on which these estimates were based is small.

\section{Association between behaviour change and changes in HIV prevalence}

Between 1994 and 2007, 11 urban ANC sites were sampled consistently. Among these, mean and median HIV prevalence peaked in 1999 at 26\% and has declined since: in 2007 mean prevalence in the same sites was 15\% (figure 2A). Between 1999 and 2005, prevalence in all sites declined and by an average amount of $30 \%$. In rural areas, eight sites were consistently 

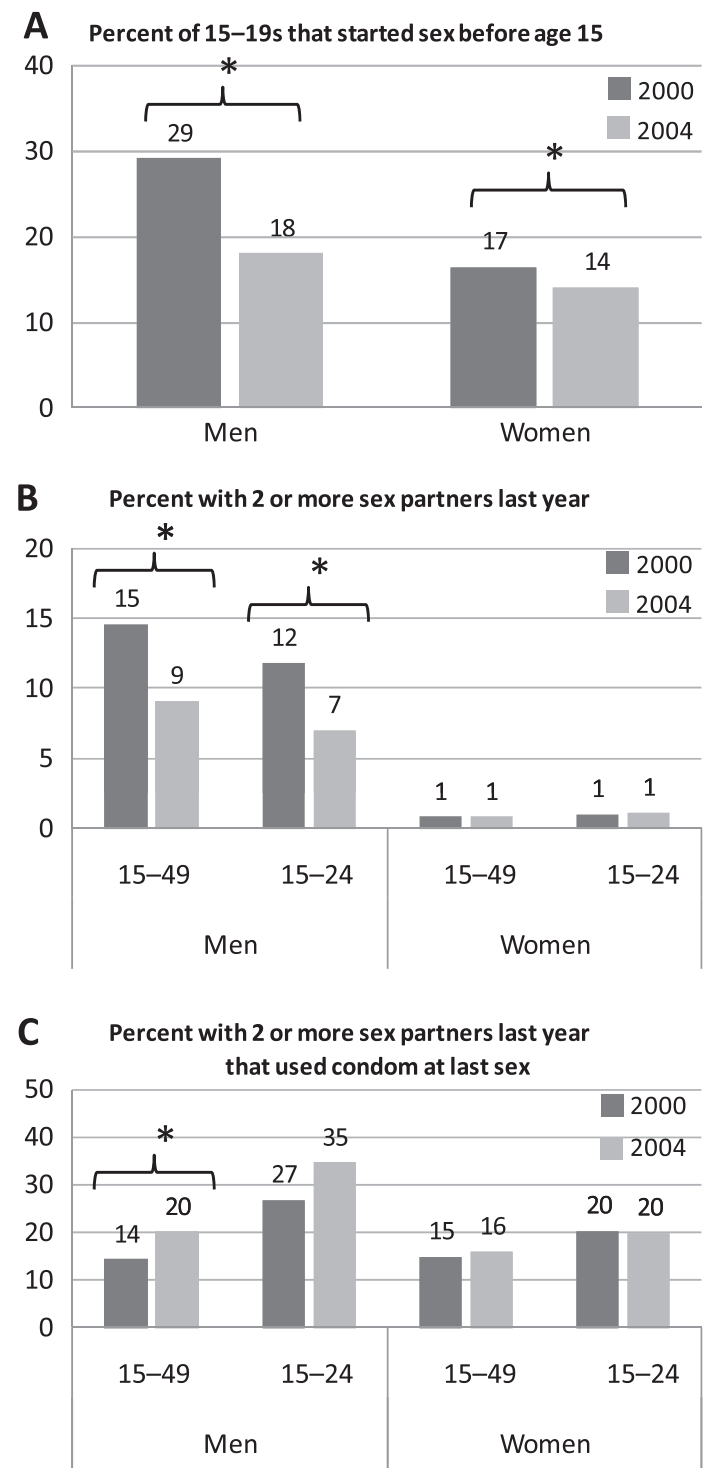

Figure 1 Changes in key sexual behaviour indicators between 2000 and 2004. Source: Malawi Demographic and Health Surveys in 2000 and 2004. The star indicates a statistically significant difference $(p<0.05)$.

included in the surveillance system. Among these, there is a less clear trend in mean and median HIV prevalence over time (figure 2B). Between 1999 and 2005, four sites indicated a decline in prevalence and four sites indicated an increase.

Among 15-24 year old women attending ANC, prevalence declined in all urban and semi-urban sites and by an average amount of $40 \%$ (figure $2 \mathrm{C}$ ). In most rural sites there was little change in prevalence (average change across sites, excluding Kasina, was $-2 \%$ ).

The model was used to test the hypothesis that the observed trends in prevalence partly result from the observed changes in sexual behaviour. In urban areas, the fit of model $M_{1}$ (with behaviour change) was superior to the fit of model $M_{0}$ without behaviour change ( $p$ value of likelihood ratio test $<0.001$ ). In addition, accounting for the information from the behavioural data in the Bayes factor analysis, there was strong evidence for behaviour change affecting the course of the epidemic (2log $(K)=19)$. Figure 3 A shows the posterior distribution for prevalence and figure $3 \mathrm{~B}$ show the posterior distribution for incidence model $M_{1}$ with a clear inflection in the period 2000-2002 where the
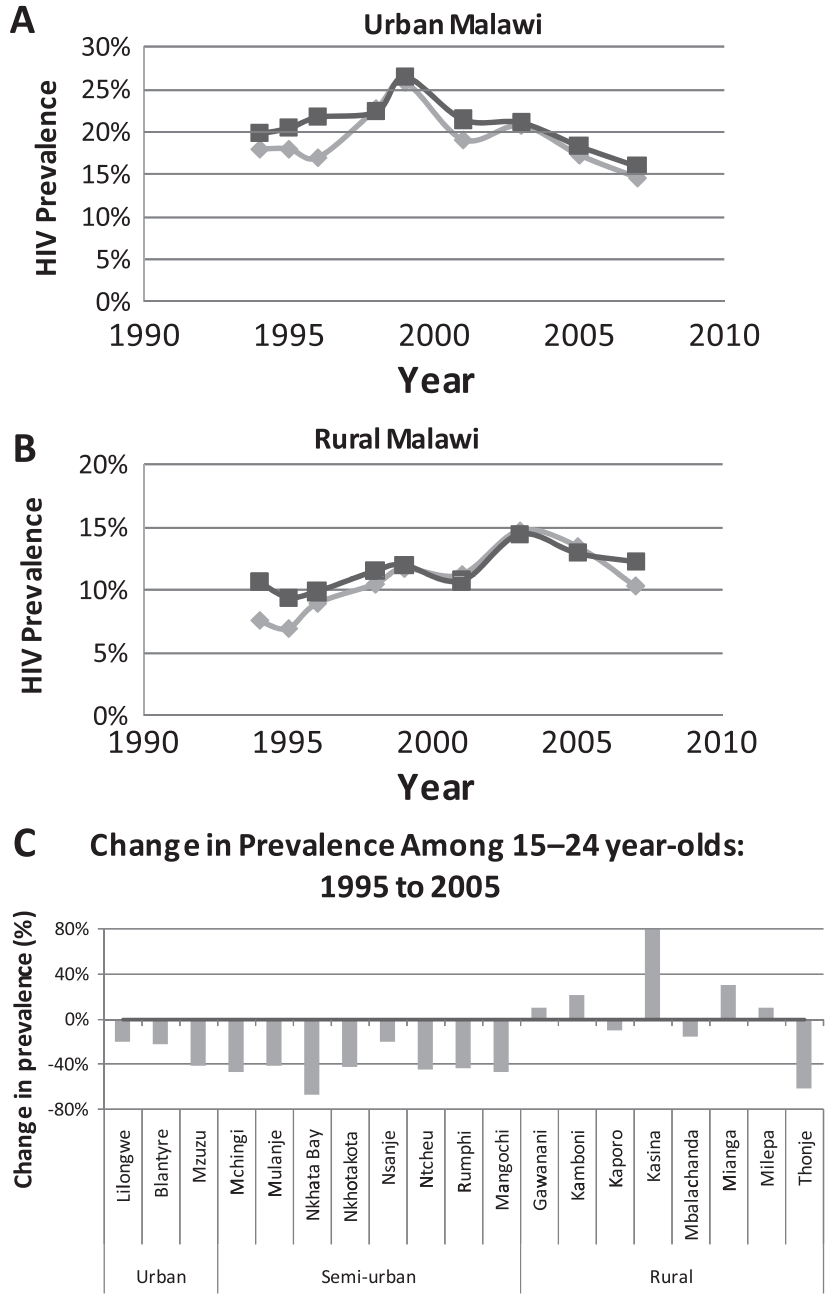

Figure 2 Trends in prevalence among consistent antenatal centres (ANC) sites in (A) urban and (B) rural Malawi; and (C) among women aged 15-24 year olds. In $A$ and $B$, the diamond line shows the median prevalence across sites; the square line shows the weighted mean prevalence across sites. In C, each bar shows the percentage change in prevalence in that ANC site between 1999 and 2005 (negative values indicate reductions). Source: Malawi National ANC Surveillance System.

impact of changes in behaviour takes effect. Table S1 online shows updated distribution for the key parameters in the analysis.

In rural areas, there was little decline in HIV prevalence and there was no evidence for behaviour changes affecting the course of epidemic in rural areas (likelihood ratio test, $\mathrm{p}=0.49$; $2 \mathrm{log}$ $(K) \approx 0$ ). Figure 3 C,D shows the posterior distribution prevalence and incidence, respectively, for model $\mathrm{M}_{0}$. Prevalence and incidence are approximately constant in the period 1999-2005, although a modest downturn in incidence is weakly supported by the data.

The number of HIV infections averted in urban areas was calculated by removing the effects of behaviour change in the posterior fits of model $\mathrm{M}_{1}$. By 2004, approximately 42000 infections were averted (95\% interval: 17000-57000). Assuming that the changes in behaviour were maintained, then in total 140000 (95\% CI: 65000 to 160000 ) infections will have been averted by 2010 .

\section{DISCUSSION}

We have presented evidence that the recent downturn in HIV prevalence in urban Malawi has partly resulted from the 
Figure 3 Model fits to the Malawi epidemic in urban and rural areas of Malawi. Panels $(A)$ and $(C)$ show the modelled HIV prevalence (\%) trend over time: the crosses show data points from individual ANC sites and the lines join data from the same clinic; the solid line show the modal model (with calibration) run in the posterior distribution (ie the run best supported jointly by the prevalence and the behaviour data) and the dashed lines show $95 \% \mathrm{Cl}$ for prevalence at each point in time from the posterior distribution. Panels (B) and (D) show the modelled HIV incidence (per 100 person-years at risk) trend over time for the general population: the solid line shows the median incidence estimate and the dashed lines show the $95 \% \mathrm{Cl}$ for incidence at each point time from the posterior distribution. The model fit shown for the urban areas is model $M_{1}$ and the model fit shown for rural areas is $M_{0}$.
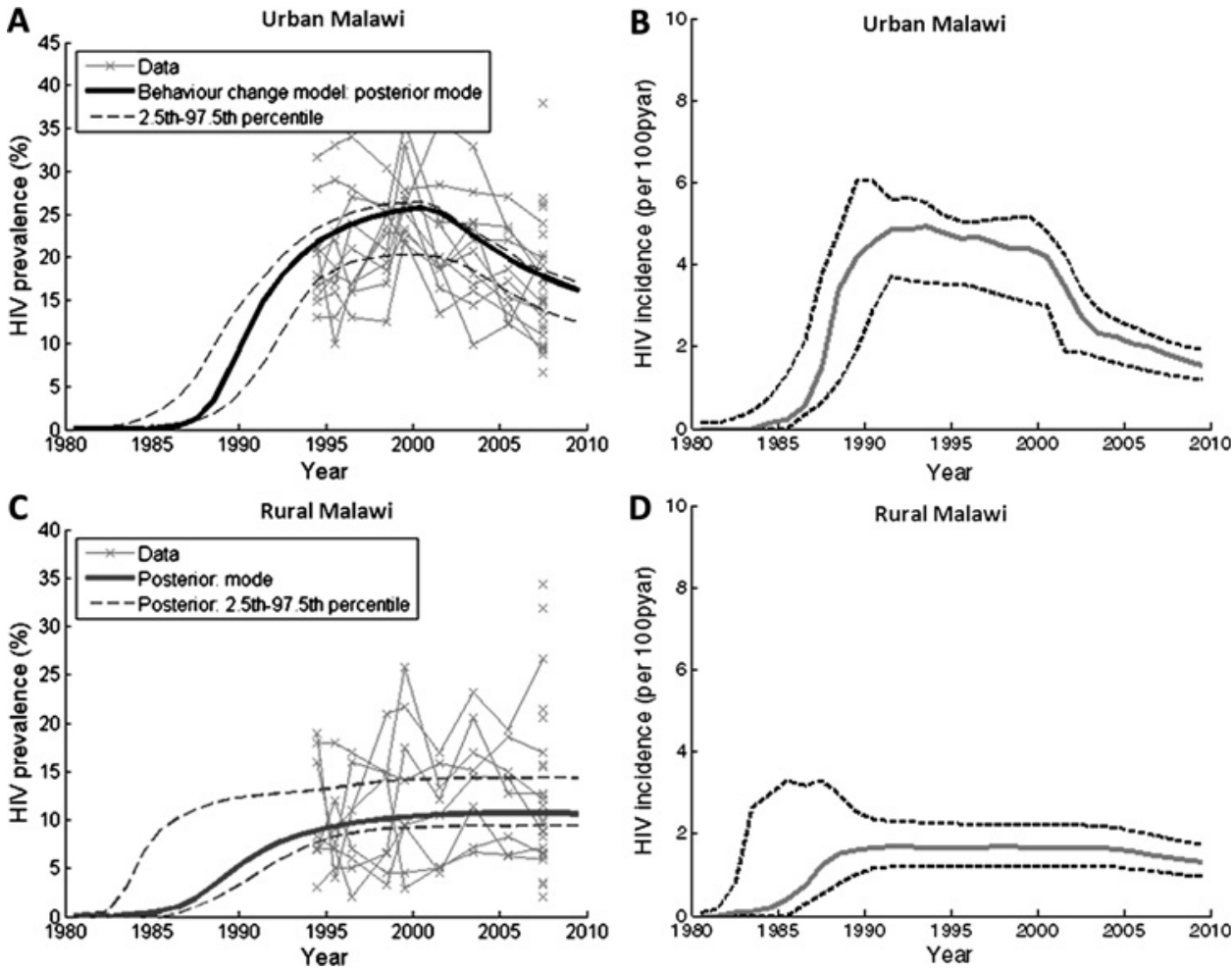

concomitantly observed changes in sexual risk behaviour, while the epidemic in rural Malawi has remained stable. The risk changes began in 2000-2002, and have averted $\sim 140000 \mathrm{HIV}$ infections by 2010, and this will lead to reduced AIDS death and antiretroviral treatment costs in the future.

The proximate factors most consistent with underpinning this reduction in risk include reductions in the proportion of men that have several sexual partners and an increase in condom use. The changes in the timing of starting sex is less likely to have played a substantial role in reducing risk overall, since these changes probably lead to only modest reductions in the lifetime and population-level risk of HIV infection. ${ }^{30}$ Recognising that the impact of changes in proximate determinants of risk can be different according to the precise nature of the epidemic context, about which we have collated limited information, it seems that the reductions in the number of men with multiple partners is more likely to have played the principal role because these changes were more substantial than the changes in condom use and, in mathematical models, large reduction in partner numbers tend to influence transmission more than small increases in condom use. ${ }^{31}$ However, an important caveat is that only basic indicator information about sexual behaviour was available and other aspects of sexual behaviour not directly recorded (eg, concurrency of partnerships, ${ }^{32}$ consistency of condom use across many sex acts ${ }^{33}$ ), which can also have an important influence of HIV transmission, could also have changed and formed a part of the difference in overall risk.

What precipitated these behaviour changes at around 2000-2002? The 2006 Malawi Triangulation Project ${ }^{34}$ reviewed published and unpublished data on the expansion of intervention programmes and found a broad correlation between the reach of interventions in regions and a downward trend in risk behaviour and HIV prevalence. That report credited increases in condom distribution and HIV testing with a primary role in driving the change in HIV incidence. However, while programmes may have equipped individuals with the information and services they needed to reduce their risk of infection, they may not necessarily have provided the impetus for behaviour change. In Malawi in the early 2000s, witnessing AIDS illnesses and deaths formed a part of everyday life for most people and it seems likely that the fear of becoming infected could motivate substantial changes in sexual behaviour ${ }^{35}$ (the 'funeral effect'). The risk reduction in Zimbabwe and Uganda has at least partly been attributed to this process. ${ }^{9}{ }^{12}$ Although the model indicates that risk reduced in the period 2000-2002, this is a simplification and it is more likely a gradual change in behaviour beginning in the late 1990s gathered pace or reach a 'tipping point' around 2000-2002 when it began to have an influence on the epidemic.

In this analysis we have focused on a selection of three key behaviour indicators that do not fully capture the range of ways that sex can lead to infection risk. The data are also collected in a face-to-survey and there is scope for social desirability biases to both understate the level of sexual risk behaviour and to generate a trend towards reporting safer sexual behaviour over time. ${ }^{36}$ The finding from the model are based on accepting that it adequately reflects HIV transmission in these populations. In particular, the erroneous conclusion that behaviour change has contributed to prevalence declines would be drawn if the simulation model does not fully capture the extent to which prevalence can decline as a natural part of the epidemic maturation. The model does not explicitly represent how the sample of women attending ANC might change as the epidemic evolves and AIDS-related sub-fertility leads to more infected women being excluded from the ANC sample. This could theoretically lead to greater declines in prevalence, but models that have examined this ${ }^{14} 2237$ find that any such change is likely to occur shortly after incidence peaks so later declines in prevalence will not be fully explained by this process.

The model also does not capture the finite duration of sexual partnerships, temporal overlaps between sexual partnerships ${ }^{32}$ or the possible range of sex partner network structures that have been shown to influence patterns of transmission. ${ }^{38}$ For instance, the model used here could underestimate declines in 


\section{Key messages}

- Between 2000 and 2004 in Malawi, substantial changes in sexual behaviour were reported, including the proportion of men with multiple sexual partners.

- In the same period, prevalence dropped from $26 \%$ to $15 \%$ in urban areas among pregnant women and reduced by $40 \%$ among women aged $15-24$ years.

- Mathematical modelling suggests that the declines in prevalence in urban areas were associated with these behavioural changes, which averted approximately $\sim 140000$ HIV infections.

prevalence if most transmission in the early phase of the epidemic is associated with a large network component supported by only a few individuals. However, models that do capture these effects tend to show much less substantial declines in prevalence than this model generates. In fact, the range of parameter values supported by the prior distribution in this model is likely to generate greater declines than could realistically be expected and will, therefore, lead to conservative conclusions being drawn about the influence of behaviour change.

In conclusion, we have shown that changes in sexual behaviour can avert thousands of new HIV infections in mature generalised hyper-endemic settings. In urban Malawi, the reduction in the number of men with multiple partners is the factor most consistent with driving the reduction in incidence. However, understanding the causes of this change is a priority, so that successful programmes and campaigns can be rapidly expanded to rural areas and other countries in the region.

\section{Acknowledgements TBH thanks the Wellcome Trust for funding support. \\ Funding Wellcome Trust}

Competing interests None declared.

Contributors All authors contributed to designing and analysing/interpreting the model and writing the manuscript. GB led the compilation of data and TBH led the modelling component.

Provenance and peer review Not commissioned; externally peer reviewed.

\section{REFERENCES}

1. Sepulveda J, Carpenter C, Curran J, et al; Committee for the Evaluation of the President's Emergency Plan for AIDS Relief (PEPFAR) Implementation. PEPFAR Implementation: Progress and Promise. Washington, D.C: Institute of Medicine of the National Academies, 2007.

2. Weiss HA, Wasserheit JN, Barnabas RV, et al. Persisting with prevention: the importance of adherence for HIV prevention. Emerg Themes Epidemiol 2008;5:8.

3. UNAIDS. Report on the Global AIDS Epidemic. Geneva: UNAIDS, 2008. http://www. unaids.org/en/KnowledgeCentre/HIVData/GlobalReport/2008/2008_Global_report. asp.

4. Hankins C, Hargrove J, Williams B, et al; UNAIDS/WHO/SACEMA Expert Group on Modelling the Impact Cost of Male Circumcision for HIV Prevention. Male circumcision for HIV prevention in high HIV prevalence settings: what can mathematical modelling contribute to informed decision making? PLoS Med 2009;6 e1000109.

5. Hallett TB, Singh K, Smith JA, et al. Understanding the impact of male circumcision interventions on the spread of HIV in southern Africa. PLOS ONE 2008;3:e2212.

6. Abbas UL, Anderson RM, Mellors JW. Potential impact of antiretroviral chemoprophylaxis on HIV-1 transmission in resource-limited settings. PLoS One 2007; $:$ : 875

7. Granich RM, Gilks CF, Dye C, et al. Universal voluntary HIV testing with immediate antiretroviral therapy as a strategy for elimination of HIV transmission: a mathematical model. Lancet 2009;373:48-57.

8. Kilian AH, Gregson S, Ndyanabangi B, et al. Reductions in risk behaviour provide the most consistent explanation for declining HIV-1 prevalence in Uganda. AIDS 1999; 13:391-8
9. Stoneburner RL, Low-Beer D. Population-level HIV declines and behavioral risk avoidance in Uganda. Science 2004;304:714-18.

10. Nelson KE, Celentano DD, Eiumtrakol $\mathrm{S}$, et al. Changes in sexual behavior and a decline in HIV infection among young men in Thailand. N Engl J Med 1996;335:297-303

11. Rojanapithayakorn W, Hanenberg R. The $100 \%$ condom program in Thailand. AIDS 1996;10:1-7

12. UNAIDS. Evidence for HIV Decline in Zimbabwe: A Comprehensive Review of the Epidemiological Data, 2005. http://www.epidem.org.

13. Hallett TB, Gregson $S$, Gonese $E$, et al. Assessing evidence for behaviour change affecting the course of HIV epidemics: a new mathematical modelling approach and application to data from Zimbabwe. Epidemics 2009:1:108-17.

14. Hallett TB, Aberle-Grasse J, Bello G, et al. Declines in HIV prevalence can be associated with changing sexual behaviour in Uganda, urban Kenya, Zimbabwe, and urban Haiti. Sex Transm Infect 2006;82(Suppl 1):i1-8.

15. Walker P, Hallett TB, White PJ, et al. Interpreting declines in HIV prevalence: the impact of spatial aggregation and migration on expected declines in prevalence. Sex Transm Infect 2008;84:iii42-8.

16. Grassly NC, Garnett GP, Schwartlander B, et al. The effectiveness of HIV prevention and the epidemiological context. Bull World Health Organ 2001;79:1121-32.

17. Hallett TB, White PJ, Garnett GP. Appropriate evaluation of HIV prevention interventions: from experiment to full-scale implementation. Sex Transm Infect 2007;83:55-60.

18. National Statistical Office [Malawi]. Macro O. Malawi Demographic and Health Survey 2000. Zomba, Malawi and Calverton, Maryland, USA: National Statistical Office and ORC Macro, 2001

19. National Statistical Office [Malawi]. Macro O. Malawi Demographic and Health Survey 2004. Calverton, Maryland, USA: National Statistical Office and ORC Macro, 2005.

20. Poole D, Raftery AE. Inference for deterministic simulation models: the Bayesian melding approach. J Am Stat Assoc 2000;95:1244-55.

21. Alkema L, Raftery AE, Clark SJ. Probabilistic projections of HIV prevalence using Bayesian melding. Ann Appl Stat 2007:1:229-48.

22. Garnett GP, Gregson S. Monitoring the course of the HIV-1 epidemic: the influence of patterns of fertility on HIV-1 prevalence estimates. Math Popul Stud 2000:8:251-77.

23. Alkema L, Raftery AE, Brown T. Bayesian melding for estimating uncertainty in national HIV prevalence estimates. Sex Transm Infect 2008:84(Suppl 1):i11-16.

24. Ghys PD, Walker N, McFarland W, et al. Improved data, methods and tools for the 2007 HIV and AIDS estimates and projections. Sex Transm Infect 2008;84(Suppl 1): i1-4.

25. Brown T, Grassly NC, Garnett G, et al. Improving projections at the country level: the UNAIDS estimation and projection package 2005. Sex Transm Infect 2006;82 (Suppl 3):iii34-40.

26. Zaba B, Boerma T, White R. Monitoring the AIDS epidemic using HIV prevalence data among young women attending antenatal clinics: prospects and problems. AIDS 2000;14:1633-45.

27. Gouws E. Comparison of HIV prevalence in population-based national surveys and ANC surveillance: implications for calibrating surveillance data. Sex Transm Infect 2008;84(Suppl 1)i17-23.

28. Jeffreys H. The Theory of Probability. New York, USA: Oxford University Press, 1961

29. Raftery A. Hypothesis testing and model selection. In: Gilks W, Richardson S Spiegelhalter D, eds. Markov Chain Monte Carlo in Practice. London: Chapman \& Hall, 1996.

30. Hallett TB, Gregson S, Lewis JJC, et al. Behaviour change in generalised HIV epidemics: impact of reducing cross-generational sex and delaying age at sexual debut. Sex Transm Infect 2007:83:i50-4.

31. Garnett GP, White PJ, Ward H. Fewer partners or more condoms? Modelling the effectiveness of STI prevention interventions. Sex Transm Infect 2008;84(Suppl 2): ii4-11.

32. Morris M, Kretzschmar M. Concurrent partnerships and the spread of HIV. AIDS 1997:11:641-8.

33. Bracher M, Santow G, Watkins SC. Assessing the potential of condom use to prevent the spread of HIV: a microsimulation study. Stud Fam Plann 2004;35:48-64.

34. National AID Commission Malawi, World Health Organization, University of California San Francisco, UNAIDS, Centers for Disease Control and Prevention. Report of the Malawi Triangulation Project: Synthesis of Data on Trends in the National and Local HIV Epidemics And The Reach and Intensity of Prevention Efforts. Process, Key Findings, and Recommendations. Lilongwe: Malawi National AIDS Commission, 2006

35. McKusick L, Wiley JA, Coates TJ, et al. Reported changes in the sexual behavior of men at risk for AIDS, San Francisco, 1982-84-the AIDS Behavioral Research Project. Public Health Rep 1985;100:622-9.

36. Gregson S, Zhuwau T, Ndlovu J, et al. Methods to reduce social desirability bias in sex surveys in low-development settings: experience in Zimbabwe. Sex Transm Dis 2002;29:568-75.

37. Clark SJ. An Investigation into the Impact of HIV on Population Dynamics in Africa. University of Pennsylvania, 2001. http://www.samclark.net/Files/Dissertation/ Samuel Clark Demography PhD 2001 Printed 2003-06-05.pdf.

38. Ghani $\overline{\mathbf{A}}$, Garnett GP. Risks of acquiring and transmitting sexually transmitted diseases in sexual partner networks. Sex Transm Dis 2000;27:579-87. 